\title{
INFLUENCIA DEL MODELO EDUCATIVO POR OBJETIVOS EN ELRENDIMIENTO ACADÉMICO DE LOS ESTUDIANTES DE LA UNIVERSIDAD NACIONAL DE INGENIERÍA
}

\author{
INFLUENCE OF THE EDUCATIONAL MODEL BASED ON \\ OBJECTIVES IN THE ACADEMIC PERFORMANCE OF \\ STUDENTS OF THE UNIVERSITY OF ENGINEERING
}

\author{
Tito RobertoVilchez Vilchez ${ }^{1}$, Jorge Santiago Chau Chau ${ }^{2}$ \\ RESUMEN
}

El presente artículo presenta una visión panorámica de la realidad académica de la educación superior en la Universidad Nacional de Ingeniería (UNI) y la influencia del modelo educativo actual en el rendimiento académico de los estudiantes. La educación es uno de los instrumentos más importantes con los que puede contar un país para asegurar su desarrollo humano y social, por lo cual desde la universidad debe haber un compromiso sincero de generar una revolución educativa que transforme, organice y articule el sistema en todos sus niveles con miras a garantizar una educación de calidad que produzca un excelente aprendizaje de la nueva generación estudiantil y que responda a las expectativas de esta generación para enfrentar los desafíos del mundo laboral contemporáneo. En este milenio, se ha evidenciado en la UNI un bajo rendimiento académico de los estudiantes, donde aproximadamente el40\% de estudiantes presenta un promedio de notas acumulado menor que diez (10) en la escala vigesimal. Este hecho llama la atención, considerando que a esta prestigiosa universidad ingresan los mejores estudiantes, de acuerdo con los datos proporcionados por la Oficina de Registro Central y Estadística de la UNI, considerando, además, que cuando postulan a esta casa de estudios, en promedio, de 8000 postulantes, ingresan solo 800 (10\%); sin embargo, en sus estudios universitarios, sus promedios de notas bordea en general de once (11) a doce (12), que es considerado un promedio bajo dentro de sus estudios en la universidad; al término de los estudios, a los egresados, se les dificulta insertarse en el mercado nacional e internacional. Más aún, en otras universidades particulares, tienen la nota trece (13) como nota mínima aprobatoria, lo que les permite culminar con una nota promedio superior e insertarse fácilmente en el mercado internacional. Este bajo rendimiento en la UNI se explica, entre otras razones, por los métodos tradicionales que se implementan en el proceso de enseñanza-aprendizaje sustentado en objetivos, como en la mayoría de las universidades públicas; sin embargo, no se han hecho los esfuerzos necesarios por cambiar esta situación, tampoco se han realizado investigaciones que den cuenta de la magnitud del problema. Por ello, es importante resaltar que la acción educativa no se basa únicamente en la emisión de normas que modifiquen el sistema de calificación o en los cambios de los planes de estudio y la malla curricular, sino, de forma trascendental, en la mejora de las técnicas didácticas y la acción orientadora, responsabilidad fundamental de los docentes, quienes requieren una formación continua y especializada. Al respecto, existe una cruda realidad en el sistema universitario: la Asamblea Nacional de Rectores (ANR) y las autoridades universitarias todavía no le brindan la importancia necesaria a la mejora de la calidad.

Palabras clave.- Calidad académica, educación sustentada en valores, métodos activos, métodos tradicionales, modelo educativo, rendimiento académico.

\footnotetext{
${ }^{1}$ Mag. Ing. De la Facultad de Ingeniería Mecánica de la Universidad Nacional de Ingeniería, ${ }^{2}$ Ing. De la Facultad de Ingeniería Mecánica de la Universidad Nacional de Ingeniería.
} 
académica, con el monitoreo adecuado relacionado directamente con la acreditación universitaria, todo ello vinculado con las profundas acciones por realizar en el camino hacia la excelencia académica.

\begin{abstract}
This article intends to present an overview of the academic reality of higher education at the National University of Engineering and the influence of current educational model in the academic performance of students. Education is one of the most important tools with which you can have a country to ensure their human and social development, so from the university must have a sincere commitment to create an educational revolution to transform, organize and articulate the system all levels in order to ensure quality education to produce an excellent learning the new generation of students, which meets the expectations of this generation to face the challenges of the contemporary world of work. In this millennium has been evidenced in the National Engineering University (UNI) poor academic performance of students, where approximately EL40\% of students have a cumulative GPA of less than ten (10) in the vigesimal scale. This fact is striking, considering that at this prestigious university entrants the best students, according to data provided by the Central Registry Office and Statistics UNI, considering that when applying for this university, on average, 8000 applicants, admitted only 800 (10\%), but in college, their grade point averages, bordering generally 11 to 12 which is considered a low average in their studies at the university, at the end of it difficult for them to enter the market and abroad. Moreover, in other private universities have footnote 13 as minimum passing grade, allowing them to finish with an average grade top and easily inserted in the international market. This poor performance in UNI explained among other reasons, traditional methods that are implemented in the teaching-learning process supported by objectives, as in most public universities, but have not made the necessary efforts to change this situation; less research has been done to realize the magnitude of the problem. Therefore it is important to note, that the educational activity is not based solely on the issue of rules modifying the rating system or changes in the curriculum and the curriculum, but transcendentally in improving teaching techniques and guiding action, fundamental responsibility of teachers, who require specialized training and, there being a harsh reality in the university system, in which the National Assembly of Rectors (ANR) and the university authorities still do not give the great importance of plain meaning of improving academic quality with adequate monitoring directly related to the university accreditation linked to the profound actions to perform on the road to academic excellence.
\end{abstract}

Keywords.- - Academic quality, values-based education, active methods, traditional methods, educational model, academic achievement.

\section{INTRODUCCIÓN}

Las instituciones públicas de educación superior enfrentan actualmente el reto de mejorar su calidad académica con recursos cada vez más escasos y, a la vez, deben hacer frente a las demandas de los nuevos contextos sociales y económicos de una sociedad globalizada. En la Universidad Nacional de Ingeniería (en adelante, UNI), donde se forman ingenieros, se ha prestado poca atención al estudio del rendimiento académico, a los modelos de enseñanza $\mathrm{y}$, siguiendo por años el modelo por objetivos y la repetición de los métodos con los cuales los profesores aprendieron las técnicas de ingeniería, arquitectura y ciencias. Las causas del fracaso estudiantil se han atribuido por largos años al mismo estudiante, liberando de responsabilidad al docente y a la universidad. El presente estudio revela que el modelo educativo centrado en el docente es una causa importante del fracaso estudiantil, por lo que el estudiante no es el único responsable.

El modelo educativo por objetivos centrado en el profesor relega a un segundo plano al estudiante. Se ha llegado a la conclusión de que la desmotivación es una causa grave del bajo rendimiento estudiantil que a su vez le ocasiona 
una baja autoestima. La investigación revela que el $72,3 \%$ de los estudiantes manifiesta que los profesores rara vez tratan a los estudiantes con amabilidad y respeto; y el $85 \%$ de los profesores encuestados señala que pocas veces tratan con amabilidad y respeto a los estudiantes. Sobre las notas reales en la aplicación de los diferentes sistemas de evaluación, se observa que con el sistema G, 74,24\% aprobaron determinados cursos; con estas mismas notas, se simuló con el sistema F, aprobando el $\mathbf{5 7 , 2 7 \%}$;se repitió la simulación con el sistema $\mathrm{H}$, resultando aprobados el 61,29\% de los alumnos. Ello demuestra la influencia que posee el sistema aplicado. Sin embargo, la selección de este sistema no ha sido materia de mayor análisis y su aplicación no responde a una estrategia formativa.

El método de la investigación se basa en las encuestas tomadas a los estudiantes y a los docentes. El instrumento aplicado a ambos revela un alto grado de coincidencia. Por ejemplo, el $72,3 \%$ de los estudiantes manifiesta que los profesores rara vez los tratan con amabilidad y respeto. Y el $85 \%$ de los docentes corrobora esa situación.

\begin{tabular}{l} 
La investigación revela que el \\
$72,3 \%$ de los estudiantes \\
manifiesta que los profesores \\
rara vez tratan a los \\
estudiantes con amabilidad y \\
respeto, y el $85 \%$ de los \\
profesores encuestados señala \\
que pocas veces tratan con \\
\hline
\end{tabular}

Como parte de la metodología para la validación de la respuesta de los estudiantes encuestados, se agrupó a estos, con base en su promedio de notas, en las siguientes categorías: Excelente, Muy Bueno, Bueno, Regular, Desaprobado y Reprobado. En los resultados de la evaluación a través de la prueba Chi-Cuadrado de Pearson, en el $100 \%$ de las encuestas realizadas a los estudiantes, el nivel de significancia fue superior al 0,05 , lo que indica que las opiniones fueron independientes de la categorización del estudiante, ya que sus opiniones fueron fuertemente similares, lo que implica que es el modelo educativo vigente el que afecta tanto a los de alto rendimiento como a los de

...los resultados de la evaluación a
través de la prueba Chi-Cuadrado
de Pearson, en el $100 \%$ de las
encuestas realizadas a los
estudiantes, el nivel de significancia
fue superior al 0,05 , lo que indica
que las opiniones fueron
independientes de la categorización
del estudiante...

bajo rendimiento. Es posible inferir que los estudiantes responden con objetividad, independientemente de su rendimiento académico.

Otros instrumentos de la investigación han sido las entrevistas a las autoridades, la evaluación participativa, la revisión bibliográfica, las estadísticas de los registros de notas, los informes académicos, la reglamentación y la revisión de trabajos pioneros en el ámbito de la formación universitaria en ingeniería de A. Padilla (2010), J. Breña (2007) y G. Huamaní (2008), profesores de la UNI.

Entre las conclusiones más reveladoras, se encuentra que el modelo actual no prioriza al estudiante, como se ha demostrado en el estudio, donde el $71,4 \%$ de las autoridades considera que los horarios de clases favorecen a los profesores, lo que coincide con el 75,86\% de la opinión de los estudiantes encuestados, pero no favorece a estos últimos.

Asimismo, el $57,1 \%$ de las autoridades reconoce que los horarios de clases no están bien confeccionados. Ello grafica una debilidad en el modelo educativo por objetivos, independiente de los gestores: el modelo prioriza al profesor y las decisiones se toman siempre a su favor.

El 100\% de las autoridades entrevistadas señala que la implantación del Modelo Educativo por Competencias es lo más adecuado y conveniente para las nuevas generaciones.

Es evidente que la comunidad universitaria (autoridades, profesores y estudiantes) están de acuerdo en la necesidad de un nuevo modelo educativo. 


\section{MARCO METODOLÓGICO}

\section{Tipo y nivel de investigación}

Nivel de investigación.- Básico. Se realiza principalmente para conocer los fundamentos de los hechos que se estudian.

Tipo de investigación.- Descriptivo- explicativo.

\section{Población y muestra}

Población.- La UNI, de acuerdo con los datos que se hallan en la Oficina de Registro Central y Estadística, cuenta con una población de 10782 estudiantes, 1267docentes y41 autoridades. Esta población universitaria comprende estudiantes, profesores y autoridades desde el punto de vista académico, que se encuentran en el campus de la UNI.

Los elementos que constituyen la población $(\mathrm{N})$ para la presente investigación son:

Estudiantes $=10872$

Profesores $=1267$

Tabla 1. Total de alumnos en pregrado

Autoridades Académicas = Rector, Vicerrectores,

Decanos, Directores de Escuela $=14$

\begin{tabular}{|c|c|c|}
\hline $\mathrm{N}=$ & FACULTAD & $\begin{array}{l}\text { CANTIDAD DE } \\
\text { MATRICULADOS }\end{array}$ \\
\hline 1 & FAUA & 897 \\
\hline 2 & FIC & 1478 \\
\hline 3 & FIECS & 917 \\
\hline 4 & FIGMM & 759 \\
\hline 5 & FIIS & 1425 \\
\hline 6 & FIEE & 1241 \\
\hline 7 & FIM & 1533 \\
\hline 8 & FC & 702 \\
\hline 9 & FIP & 392 \\
\hline 10 & FIQT & 885 \\
\hline \multirow[t]{2}{*}{11} & FIA & 553 \\
\hline & TOTAL: & 10782 \\
\hline
\end{tabular}

$10872+1267+14=12180$, entonces: $\mathrm{N}=12153$

Nivel de confiabilidad para la muestra: $95 \%$. $\varepsilon=5 \%$, es el margen de error estimado.
Proporción de la población con mayor grado de relación o conocimiento con el problema del bajo rendimiento académico estudiantil en la Universidad Nacional de Ingeniería: probabilidad de éxito $\mathrm{p}=0,5$, que es equivalente al $50 \%$.

Proporción de la población con menor grado de relación o conocimiento del problema del bajo rendimiento académico estudiantil en la UNI: probabilidad de fracaso $\mathrm{q}=0,5$, que es equivalente al $50 \%$.

$\mathrm{Z}=1,96$, que es el valor normal estándar correspondiente al nivel de confianza de $95 \%$. Por ser una población finita ( $\mathrm{N}=12153$ personas), la muestra se determinará mediante la Técnica de Muestreo Proporcional, a través de la siguiente fórmula:

$$
n=\frac{Z^{2} p q N}{\varepsilon^{2}(N-1)+Z^{2} p q}
$$

Remplazando los valores numéricos correspondientes, el tamaño de la muestra mínima que es posible utilizar para la investigación debe ser:

$$
n=\frac{1.96^{2}(0.5)(0.5) 12180}{0.050^{2}(12180-1)+1.96^{2}(0.5)(0.5)}=373 \text { personas }
$$

\section{Estratificación de la muestra}

Los subgrupos o estratos que se consideraran son los siguientes: estudiantes, profesores y autoridades. Para distribuir la muestra total $(\mathrm{n}=$ 373 personas) en cada uno de los estratos mencionados, se determina la proporción correspondiente, aplicando la siguiente fórmula:

$$
n_{\text {sub }}=\frac{N_{h}}{N}(n)
$$

Donde $\mathrm{N}_{\mathrm{h}}=$ Subpoblación

$\mathrm{N}=$ Muestra de la población total

$\mathrm{n}_{\text {sub }}=$ Muestra representativa del subgrupo

Muestra representativa del estrato o subgrupo de estudiantes:

$$
n_{\text {est }}=\frac{10872}{12180}(373)=333
$$


Entonces el estrato de estudiantes es de 333. Muestra representativa del estrato o subgrupo de profesores:

$$
n_{\text {prof }}=\frac{1267}{12180}(373)=38
$$

Entonces, el estrato de profesores es de 38. Por ser muy baja la cantidad de profesores, se tomará en forma independiente un estrato representativo de la población.

Muestra representativa del estrato o subgrupo de autoridades académicas como mínimo será de:

$$
n_{\text {autor }}=\frac{41}{12180}(373)=2
$$

Sin embargo, se tomará una muestra de 7 autoridades con la finalidad de que sea más representativa.

Comprobación de la representatividad de la muestra:

$\mathrm{n}=\mathrm{n}_{\text {est }}+\mathrm{n}_{\text {prof }}+\mathrm{n}_{\text {autor }}$

$\mathrm{n}=333+38+2=373$ personas como [Aquí falta completar la expresión].

\section{TÉCNICAS E INSTRUMENTOS DE RECOLECCIÓN DE DATOS}

\section{Técnicas para recolectar Información}

a) Análisis de documentos.- Consistió en el estudio detallado de los diversos documentos, ya sean técnicos pedagógicos y estadísticos, para obtener una información valida sobre los diversos aspectos evaluados, que serán analizados en función a los objetivos de la investigación.

b) Documentos normativos.- Se estudió las normas académicas relacionadas con el modelo educativo tradicional de la universidad, la Ley General de Educación vigente ( $\left.\mathrm{N}^{\circ} 28044\right)$, la Ley del Sistema universitario $\left(\mathrm{N}^{\circ}\right.$ 23733), los documentos normativos de la UNI como el Estatuto de la Universidad, el Reglamento Académico y otras normas universitarias vigentes. c) Documentos técnicos pedagógicos.- La fuente de datos más importantes de las dimensiones de contexto y procesos de los documentos técnico pedagógicos fue la elaborada en la universidad: currículos, sílabos, normas, así como informes de las evaluaciones realizadas a nivel de la UNI.

d) Información estadística.- Esta información se obtuvo de la Oficina de Registro Central y Estadística de la Universidad Nacional de Ingeniería.

e) La encuesta.- Esta técnica se utilizó para la obtención de datos de la muestra de los alumnos y profesores. Fue elaborada para determinar el nivel de eficacia de los medios en la formación de los estudiantes de la universidad.

f) Entrevistas.- Esta técnica se utilizó como prueba para recolectar información de las autoridades de la universidad.

\section{Instrumentos}

Cuestionarios.- Se aplicó como prueba tres cuestionarios, dirigidos a los estudiantes, profesores y autoridades para recabar la información acerca de la gestión de la calidad educativa, sobre el modelo educativo, la metodología, las estrategias y los contenidos educativos.

\section{Técnicas para el procesamiento de datos}

Entre las principales técnicas que se aplicó en este estudio, tenemos:

Medidas de posición y de dispersión, así como pruebas de significación del estimador de tendencia central y dispersión.

\section{Técnicas para la prueba de hipótesis}

La contrastación de las hipótesis se realizó con la prueba paramétrica llamada Chi Cuadrado, con la finalidad de determinar si existe o no asociación entre las variables. La información recopilada fue procesada a través del paquete estadístico SPSS versión 15.

\section{PRINCIPALES RESULTADOS}

Las figuras que se presentan a continuación revelan los principales resultados: 


\section{La teoría no se vincula a casos reales}

El modelo acerca de la enseñanza no vincula la teoría con problemas de casos reales. $\mathrm{La}$ percepción estudiantil revela que solo un 47,75\% de los profesores trata en clase la solución de problemas con casos de la vida real.

\section{El profesor promueve la solución de problemas con casos de la vida real?}

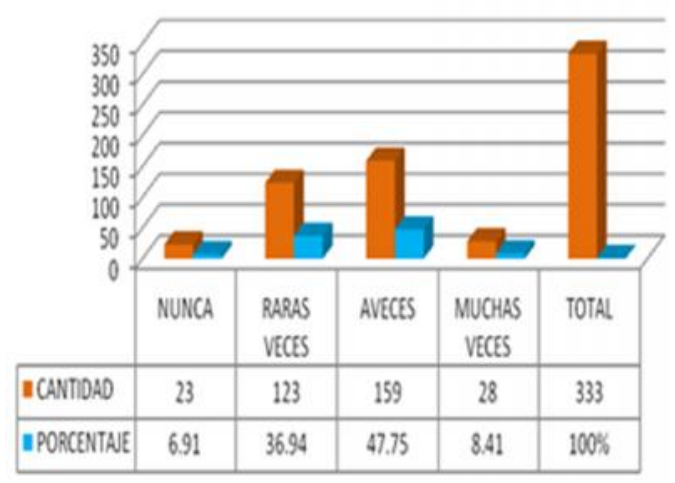

Fig. 1 Resultados a la pregunta: ¿El profesor promueve la solución de problemas de la vida real?.

Ref. ${ }^{1}$ Figura 4.68. Figura sobre valoración estudiantil 2

\section{METODOLOGÍA ACADÉMICA}

En este aspecto, la investigación revela que el método tradicional no considera la opinión del estudiante.

Más del $49 \%$ indican que el profesor no toma en cuenta la opinión estudiantil y, además, no existe trato adecuado entre ambos.
Consideras que el profesor toma en cuenta la opinión de los estudiantes?

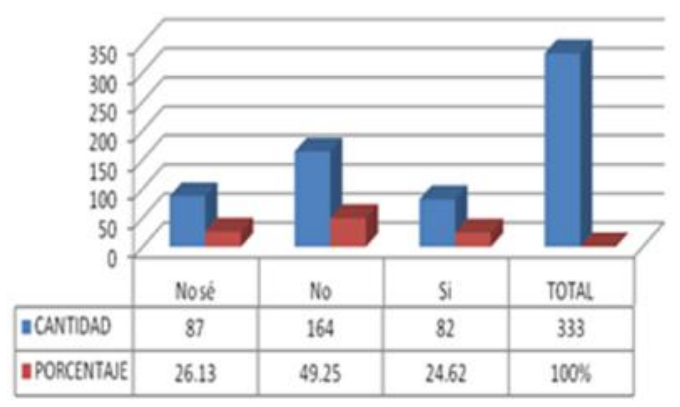

Fig. 2 Resultados a la pregunta: ¿Consideras que el profesor toma la opinión de los estudiantes?

Figura $^{2}$ 4.107. Figura sobre valoración estudiantil 9.

\section{Contenidos alejados de las competencias requeridas en el entorno laboral}

Entre las competencias que exige el mercado laboral en la actualidad, se encuentran el trabajo en equipo, multidisciplinario y multicultural; es lógico esperar que sea una de las actividades importantes en la formación profesional. Sin embargo, el estudio revela que más del $65 \%$ de los profesores no promueve el trabajo en equipo.

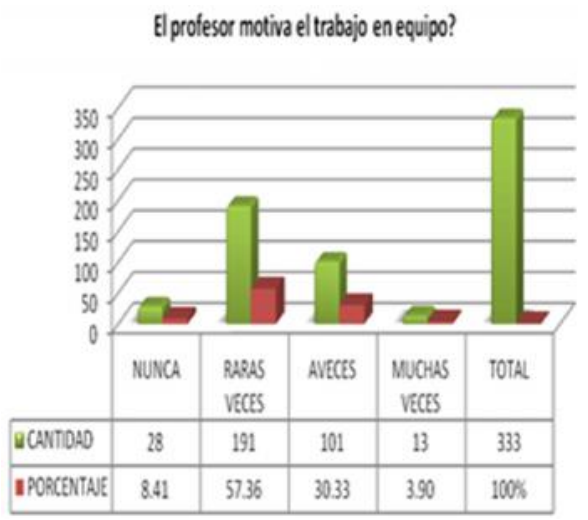

Fig. 3 ¿El profesor motiva el trabajo en equipo?.

Figura4.117. Cuadro de valoración estudiantil 19

\footnotetext{
${ }^{2}$ La numeración indicada está referida al documento original del informe de la investigación presentada al IGI.
}

\footnotetext{
${ }^{1}$ La numeración indicada está referida al documento original del informe de la investigación presentada al IGI.
} 
Sobre los contenidos académicos que el profesor proporciona, el estudiante manifiesta que se encuentra poco satisfecho, un $90 \%$ de los estudiantes encuestados manifiesta que no están totalmente satisfechos.

\section{Cuán satisfecho (a) te encuentras con los contenidos que proporciona el profesor?}

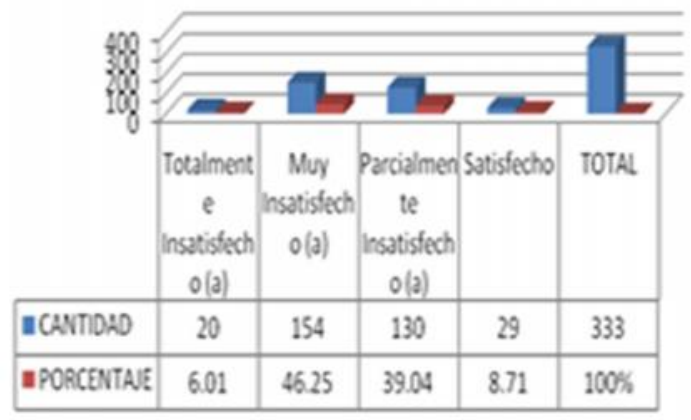

Fig. 4 ¿Cuán satisfecho (a)te encuentras con los contenidos que proporciona el profesor?.

\section{La segmentación de la población estudiantil} encuestada por el rendimiento académico

Existe gran confiabilidad de los resultados de las encuestas. La población segmentada no revela distorsiones por la condición de aprobado o desaprobado en sus respuestas. Ante la pregunta ¿el profesor propicia la participación de los estudiantes?, se observa que los porcentajes de respuestas por alternativa son estadísticamente iguales.

Tabla 2. Total de docentes en pregrado.

\begin{tabular}{|ll|c|c|c|c|c|}
\hline & & Nunca & Rara vez & Aveces & muchas veces & Total \\
\hline Reprobado & Recuento & 24 & 130 & 73 & 12 & 229 \\
& \% & $10.5 \%$ & $56,8 \%$ & $27.5 \%$ & $5.2 \%$ & $100.0 \%$ \\
\hline Desaprobado & Recuento & 101 & 537 & 244 & 56 & 938 \\
& $\%$ & $10.8 \%$ & $57.2 \%$ & $26.0 \%$ & $6.0 \%$ & $100.0 \%$ \\
\hline Aprobado & Recuento & 198 & 944 & 440 & 92 & 1674 \\
& \% & $11,8 \%$ & $56.4 \%$ & $26,3 \%$ & $5.5 \%$ & $100.0 \%$ \\
\hline Bueno & Recuento & 178 & 966 & 483 & 102 & 1729 \\
& \% & $10.3 \%$ & $55,9 \%$ & $27.9 \%$ & $5.9 \%$ & $100.0 \%$ \\
\hline Excelente & Recuento & 2 & 16 & 8 & 2 & 28 \\
& \% & $7,1 \%$ & $57,1 \%$ & $28.6 \%$ & $7,1 \%$ & $100.0 \%$ \\
\hline Total & Recuento & 503 & 2593 & 1238 & 264 & 4598 \\
& \% & $10.9 \%$ & $56,4 \%$ & $26.9 \%$ & $5,7 \%$ & $100.0 \%$ \\
\hline
\end{tabular}

Ref. Tabla A.46 Tabla de Aprobados y Desaprobados 21.

Un aspecto sorprendente es el que corresponde al estudiante que revela que no existe un buen trato del docente hacia el estudiante y la encuesta en los docentes la ratifica y además las autoridades también lo reconocen.

\section{El profesor trata a los estudiantes con amabiidad yrespetto?}

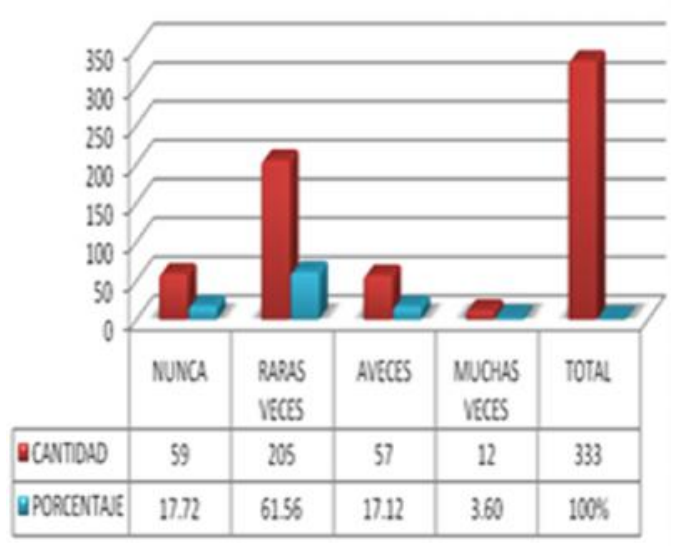

Fig. 5 ¿El profesor trata a los estudiantes con amabilidad y respeto?

\section{CONCLUSIONES}

Es preciso destacar que todas estas conclusiones deben ser tomadas con mucha cautela. El contexto y las circunstancias en los que se ha llevado a cabo la investigación establecen los límites entre los resultados y la realidad. La población es amplia, la muestra es concreta. Algunos errores de medida se asumen partiendo de que los evaluados son personas diferentes, autoridades, docentes y estudiantes.

En el $100 \%$ de las encuestas realizadas a los estudiantes, el nivel de significancia fue superior al 0,05 , lo que indica que las opiniones fueron independientes de la categorización del estudiante, ya que sus opiniones fueron fuertemente similares. Ello implica que es el modelo educativo vigente el que afecta tanto a los de alto rendimiento como a los de bajo rendimiento, como se evidencia en la Tabla 2.

En definitiva, la conclusión fundamental es que la mayoría de las variables que discriminan entre los 
estudiantes de rendimiento bajo y de los demás estudiantes están en manos del modelo educativo.

El 71,4\% de las autoridades entrevistadas reconoce que el modelo educativo actual, vigente por más de seis décadas, no ha sido cambiado por desconocimiento y falta de voluntad política. El $100 \%$ de las autoridades entrevistadas señala que la implantación del Modelo Educativo por Competencias es lo más adecuado y conveniente para las nuevas generaciones.

Es evidente que la comunidad universitaria (autoridades, profesores y estudiantes) están de acuerdo en la necesidad de un nuevo modelo educativo.

El diagnóstico del trabajo de investigación concluye lo siguiente:

El resultado negativo del Modelo Tradicional se hace patente cuando el estudio revela que el $73 \%$ de los estudiantes encuestados no se sienten preparados para asumir las exigencias de la sociedad y del mercado ocupacional.

El modelo educativo tradicional por objetivos tiene influencia negativa en el rendimiento académico de los estudiantes.

Se demuestra comprobando que:

Los planes de estudio vigentes en el $74 \%$ de las escuelas profesionales se encuentran desactualizados, lo que impide la competitividad de los egresados de la UNI ante el mercado internacional. Según documentos oficiales, los planes de estudio tienen más de dos años de antigüedad y sus equivalencias no están actualizadas, no acordes con los actuales requerimientos del mercado laboral, lo que constituye una fuerte debilidad en la gestión curricular.

El modelo educativo por objetivos está obsoleto y mal enfocado, dado que solo se concentra en objetivos educativos centrados en el profesor, que se evidencia, por ejemplo, cuando el $63 \%$ de los estudiantes manifiesta que el profesor rara vez motiva el trabajo equipo. En la actualidad, se exige en forma prioritaria el trabajo colaborativo como se señala en el Proyecto Tuning y las instituciones acreditadoras de las carreras universitarias.
El modelo educativo por objetivos tiene fuertes debilidades, dado que no mide logros educativos; solo ejecuta una evaluación o calificación de las prácticas y/o exámenes fría, sin diagnósticos de causa y efecto, por lo cual es necesario que los resultados de la evaluación docente se deban evidenciar con logros educativos por parte del profesor a través de sus indicadores de logros, según la observación participante.

El modelo educativo no señala, en los planes de estudio, estrategias de retroalimentación ni pautas para la evaluación continua e integral.

La hipótesis sobre la influencia negativa de las estrategias académicas del modelo educativo tradicional, queda demostrada a través de:

Las estrategias educativas y contenidos académicos requieren de actualización; el 73\% de los estudiantes manifiesta que el profesor no utiliza las tecnologías de la información y la comunicación (en adelante, TIC)en el proceso de enseñanza, siendo en la actualidad una exigencia del mundo laboral. De acuerdo con la observación participante en 200 muestras, se evidenció que solo el 35\% utilizaba tecnologías TIC en forma adecuada.

El $66 \%$ de los estudiantes manifiesta que el profesor no propicia la participación de los estudiantes en clases. Asimismo, el 55\% de los estudiantes señala que el profesor rara vez planifica las sesiones de aprendizaje, lo que evidencia que los profesores de la universidad en su mayoría, dada su condición de ingenieros, arquitectos y científicos, al haber ingresado a la docencia solo fueron evaluados en los aspectos cognitivos referidos a su profesión y dictan las materias de acuerdo con sus propios pensamientos, sin seguir ningún modelo educativo. lo que evidencia que carecen de fundamentos pedagógicos en la enseñanza.

Los resultados de esta investigación concluyen en que se requiere un modelo educativo centrado en el estudiante que, desde aspectos administrativos como la elaboración de horarios de clases, se hagan con criterios pedagógicos. Asimismo, los sistemas de evaluación deben cumplir función formativa $\mathrm{y}$, sobre todo, debe existir un trato respetuoso, amable y amical entre profesores y estudiantes. 
El nuevo modelo educativo en la UNI debe ser basado en la metodología activa, en la que el estudiante sea el centro del aprendizaje de las materias. Dicho modelo educativo debe formar y propiciar competencias profesionales para la vida laboral inmediata.

\section{REFERENCIAS}

1. Artunduaga M.M., "Variables que influyen en el rendimiento académico estudiantil”, Madrid España, (2008).

2. Bain, K., "Lo que hacen los mejores profesores universitarios". Valencia: Publicaciones de la Universidad de Valencia, (2006).

3. Barnett, R. "Los límites de la competencia. El conocimiento, la educación superior y la sociedad". Barcelona: Gedisa, (2001).

4. Bloom, B. "Taxonomía de los objetivos de la educación. La clasificación de las metas educacionales". Argentina: Editorial El Ateneo, Buenos Aires,(1990).

5. Boersma, Chapman "Academic selfconcept achievement expectations and focus of control in elementary learning disabled children". Canadian Journal of Behavioural Science. (1981).

6. Breña Ore, J., "Estrategias de aprendizaje y enseñanza relacionadas con el rendimiento académico de los estudiantes de Química orgánica de la facultad de Ingeniería Química y textil de la UNI". Peru (2007).

7. Callahan R. E., "Education and the Cult of Efficiency". USA(1962).

8. Clarck G., "Journal of Multicultural and Cross-Cultural Research in Art Education", 6(1), USA (1998).

9. Cruz C. J., "Razón práctica y utopía". Pamplona, ESPAÑA. (1983).

10. De La Cruz, Mª́., "Didáctica de la lección magistral". Madrid: INCIE, (1981).

11. De La Cruz, M ${ }^{a}$ Á. "Un modelo de lección magistral para un aprendizaje activo y cooperativo. Cursos y conferencias de innovación y desarrollo docente. Vigo (2004).
12. Delors J., "La educación encierra un tesoro, México, ediciones el correo de la UNESCO. (1998).

13. De La Cruz, M A., "Taller sobre el proceso de aprendizaje-enseñanza de competencias". Zaragoza: Instituto de Ciencias de la Educación. Universidad de Zaragoza.(2005).

14. De Miguel, M., "Modalidades de enseñanza centradas en el desarrollo de competencias. Oviedo: Ediciones de la Universidad de Oviedo". Estudio realizado con la ayuda del Proyecto EA2005-0118 de la Dirección General de Universidades del Ministerio de Educación y Ciencia, convocatoria. (2005-2006).

15. Fernández, A., "Formación pedagógica y desarrollo profesional de los profesores de universidad: análisis de las diferentes estrategias". Revista de Educación. Núm.331. (2003).

16. Fernández, A. "Metodologías activas para la formación de competencias" March 56 Educación siglo XXI, 24 (2006).

17. Guevara G. B., "Evaluación Constructivista", PERU, (1993).

18. Gonzales, A. H., "Taxonomía Curricular. Serie: Formación Pedagógica”. Coahuila. (1979).

19. ICE. "Guía Docente de la UPV: criterios para su elaboración. Valencia: Editorial UPV", (2006).

20. Jayawickramarajah, P. T., "Problems for Problem-Based Learning: A Comparative Study of Documents". Medical Education, (1996).

21. Lasnier, R., Réussir la formation par compétences. Montréal: Guérin.(2000)

22. Martínez Mut, B., Fernández, A., y otras autoras. "El cambio de cultura docente y el Espacio Europeo de Educación Superior", (2005).

23. Martínez, Mut., Buxarrais, M. R., Esteban, F. "La universidad como espacio de aprendizaje ético". Revista Iberoamericana de Educación, (2002).

24. Martínez Mut, B., "El perfeccionamiento del profesorado. Estrategias y modalidades de realización". Madrid: Editorial Anaya, (1983).

25. Monereo, C., Pozo, J. I., "La universidad ante la nueva cultura educativa". Madrid: Síntesis.(2003). 
26. Morales, J. J., "A evaluación en el área de educación visual y plástica en la ESO". Universidad Autónoma de Barcelona, España. (2001).

27. Moreno G., "Calidad Docente y su Influencia en la Formación Profesional del Estudiante de la Escuela Profesional de Ingeniería Acuícola de la Universidad Nacional Federico Villarreal”. Lima, Perú (2006).

28. O'Connor D. T., "E-Learning for educator: The Acorn that grew the online graduate Certificate program at UW Stout" USA.(2005).

29. Paciano Fermoso E., "Teoría de la Educación”. México (2005).

30. Padilla R. A., "Autoevaluación y gestión de la calidad hacia la acreditación de la Carrera profesional de Ingeniería Mecánica en la Universidad Nacional de Ingeniería". Perú (2010).

31. Perry\&Dickense, "Efective teaching in higher education: research and practice USA" (1984)

32. Ramsden, P., "Learning to Learn in Higher Education”. New York: Rouledge, (1993).

33. Sharan, Y., Sharan, S., "Group Investigation in the Cooperative Classroom.S.Sharan (ed.) Handbook of Cooperative Learning Methods. Westpot, CT: Greenwood Press, (1994).

34. Shmuck, R., "Learning to cooperate, cooperate to learn: Basic Concepts. R. Slavin" (ed.) Learning to cooperate, cooperating to learn. Nueva York: Plenum, (1985).

35. Slavin, R. E., "When and why does Cooperative Learning increase Achievement? Theoretical and Empirical Perspective. En R. Hertz-Lazaro witzand N. Miller (eds.) Interaction in Cooperative Groups. The Theoretical Anatomy of Group Learning. New York: Cambridge University Press, (1992).

36. Taborga H., "Reflexiones sobre planeación prospectiva de la educación superior en México". En: ANUIES. Dos décadas de planeación de la educación superior, México, (1992)

37. Tanner L. N., "La disciplina y la enseñanza en el aprendizaje”. USA (1980)
38. Turner V., "Performing Pedagogy: Toward an Art of Politics" - USA (1986)

39. UACH "La Reforma y la Innovación Curricular. Reforma Académica, un Nuevo Modelo Educativo", Dirección Académica.(2000)

40. UNESCO. "World Declaration on Higher Education for the Twenty-First Century: Vision and Action. Adopted by the World", Conference on Higher Education, Paris, FRANCIA (1998).

41. Valcárcel, "La preparación del profesorado universitario español para la Convergencia Europea en Educación Superior". Proyecto EA20030040.ESPAÑA.(2003).

42. Van keer, A., Geerlings, P., Eisendrath, H., "An Interactive Working Groupin Chemistry used as Diagnostic Tool for Problematic Study Styles". U. Chem. Ed., vol. $8, \mathrm{n}^{\circ} 1$ (2004).

43. Wellington, J., "Re-thinking the Role of Practical Work in Science Education". En Sequeira, M., Dourado, L., Vilaça, M. T., Silva, J. L., Alfonzo, A. S. y Baptista, J. Trabalho Prático e Experimental na Educaçao em Ciencia. Brafa: Universidades do Minho, (2000).

44. Yockey K. M., "Learning to write, writing to learn: theory and research in practice USA" (1998).

45. Zabalzam. A., "Competencias docentes del profesorado universitario". Madrid:Narcea ESPAÑA. (2003).

Correspondencia: titovilchez@uni.edu.pe 